\title{
ON THE DEGREE OF DISTORTIONS UNDER SECOND-DEGREE PRICE DISCRIMINATION
}

\author{
Ram Orzach
}

Oakland University, USA

orzach@oakland.edu

\section{Miron Stano}

Oakland University, USA

stano@oakland.edu

\begin{abstract}
This paper highlights the limitations and applicability of results developed by Chao \& Nahata (2015) for nonlinear pricing. Although Chao and Nahata appear to provide necessary and sufficient conditions for general utility functions, we show that one of their results leads only to a restatement of two constraints, and another result may not be valid when consumers can freely dispose of the good. Their model allows for the possibility that higher quantities will have a lower price than smaller quantities. We provide conditions under free disposal that preclude this anomaly. Our analysis suggests that further research on violations of the single-crossing condition should be encouraged.
\end{abstract}

Keywords: Second-degree price discrimination, nonlinear pricing, singlecrossing condition.

JEL Classification Numbers: D42, D61, D82, L12.

\section{INTRODUCTION}

$A \begin{aligned} & \text { N extensive literature builds on Maskin \& Riley's (1984) characterization } \\ & \text { of nonlinear monopoly pricing. }{ }^{1} \text { Maskin and Riley consider the general }\end{aligned}$ We are grateful to the editor and two anonymous referees for their many helpful comments and suggestions. The usual disclaimer applies.

1 Under nonlinear pricing, also known as a nonlinear tariff, a seller with monopoly power price discriminates by offering a menu of bundles with varying quantities and prices where the

Copyright (C) Ram Orzach, Miron Stano / 6(1), 2021, 101-112. 
case involving $\mathrm{N}$ types of consumers with differing demand functions where the monopoly seller cannot identify the individual's consumer type. The technique to maximize profits under nonlinear pricing, for the simplified example of two consumer types, is one where the firm offers two bundles of quantity and price that satisfy the incentive compatibility $(I C)$ and individual rationality $(I R)$ constraints. The $I C$ constraints require that neither type will pretend to be the other type and buy the other's quantity; while $I R$ requires that consumers buy the product only if their utilities from the exchange are non-negative.

Tirole (1988)(p.149) provides a succinct description of the relevant MaskinRiley results for two consumer types: low demand and high demand. The firm will have an optimal tariff where: i) the low demand consumer has zero surplus while the high demand has a positive one; ii) the high demand consumer will be indifferent between buying his own designated bundle and the low one (therefore, he will buy his own); and iii) the high demand type buys a quantity that corresponds to marginal cost, while the low demand consumer buys the designated quantity that is less than the one corresponding to marginal cost.

Those results are achieved under the assumption of monotonicity in utilities, a property that is known as the Sorting Condition, the Spence-Mirrlees Condition, or the Single-Crossing Condition (SCC). ${ }^{2}$ We will use SCC because the term is more revealing in that the utility functions cross only once. The SCC will be satisfied if the demand curves lie one above the other, i.e., if, at any given price, the quantity for the high demand consumer will be larger than that of the low demand consumer. In cases where the demand curves cross, as in Figure 1 in Section 2.3, the SCC may be violated.

Although applications that satisfy the SCC dominate the nonlinear pricing literature, analyses of nonlinear tariffs when the SCC is violated have appeared. Andersson (2005) develops conditions under which relaxation of the SCC will still produce the Maskin-Riley results, but Andersson (2008) further provides a counterexample to the main Maskin-Riley result. In his counterexample, the quantities for both types can correspond to those under marginal-cost pricing with zero gains for both consumer types. Chao \& Nahata (2015) (hereafter $\mathrm{CN}$ ) also consider a violation of the SCC to determine the regions in which

per-unit price typically diminishes as the bundle quantity increases. These bundles are often seen as providing quantity discounts. Nonlinear pricing has long been a fixture for many products and services.

2 See Tirole (1988)(p.148, fn 28). Within the context of this paper, any distinctions among the three conditions are not relevant. 
the quantities correspond to the efficient quantities (marginal-cost pricing) or exceed (fall short) of the efficient quantity. Our work will concentrate only on the case where the two quantities correspond to marginal cost.

$\mathrm{CN}$ appear to provide necessary and sufficient conditions for general utility functions. After describing their model in Section 2.1, Section 2.2 shows that their Proposition 2(ii) follows from a well-known mathematical method that leads only to a restatement of two constraints. Furthermore, CN's Proposition 3 provides conditions for efficient quantities, but we use a numerical example in Section 2.3 to illustrate that a portion of the proposition will have limited economic relevance when consumers can freely dispose of the good. In particular, CN's model can generate a pricing scheme where the larger quantity has a lower price than the smaller quantity. With Proposition 3 as a special case of Proposition 2, the same anomaly will also apply to Proposition 2.

To deal with this limitation, Section 3 develops an alternative to CN's Proposition 3(ii) where consumers can freely dispose of the good. In that section, we impose the conditions on the demand functions that would enable the firm to offer two menu quantities and corresponding prices so that profits are maximized and the consumer surplus is zero. The conditions also guarantee that the larger quantity will always have a higher (or equal) price than the smaller quantity a result that does not necessarily hold in the $\mathrm{CN}$ model.

\section{ARGUMENTS}

\subsection{The CN Model}

A monopolist serves two consumer types: Type $i$ utility from quantity $q$ is $u_{i}(q)$, $i \in\{1,2\}$. The seller cannot a priori distinguish between the types but knows their utilities and ratio $\gamma=n_{2} / n_{1}$ where $n_{i}$ is the number of Type $i$ consumers. Under the Revelation Principle, the monopolist can maximize profits by using nonlinear tariffs with $\left(q_{i}, T_{i}\right), i \in\{1,2\}$, where $T_{i}$ is the tariff for quantity $q_{i}$. The firm has a linear cost function with marginal cost $c \geq 0$. CN state that, without any loss of generality, the tariffs and the utilities can be normalized by the marginal cost $c$ so that $t_{i} \equiv T_{i}-c \cdot q_{i}$, and $v_{i}(q) \equiv u_{i}(q)-c \cdot q$.

With $t_{i}$ as CN's net-of-cost tariff and $v_{i}(q)$ as their net-of-cost valuation, the model assumes that $v_{i}(q)$ has a unique optimization: $q_{i}^{e} \equiv \arg \max _{q} v_{i}(q) \in$ $(0,+\infty)$, where $q_{i}^{e}$ is their efficient quantity for the Type i. The following is 
CN's constrained maximization problem (p. 209).

$$
\begin{array}{clc}
\max _{\left(t_{i}, q_{i}\right), i=1,2} & t_{1}+\gamma \cdot t_{2} & ([\mathrm{P}]) \\
\text { s.t. } & v_{i}\left(q_{i}\right)-t_{i} \geq v_{i}\left(q_{j}\right)-t_{j}, i, j=1,2 & \left(\mathrm{IC}_{i}\right) \\
& v_{i}\left(q_{i}\right)-t_{i} \geq 0, i=1,2 & \left(\mathrm{IR}_{i}\right)
\end{array}
$$

Although not defined by $\mathrm{CN}, I C$ and $I R$ represent the incentive compatibility and individual rationality constraints described in our introduction. $I C$ requires that neither consumer type will pretend to be the other type and buy the other's quantity; while $I R$ requires that each type buy his respective bundle only if the utility resulting from the purchase is non-negative. With marginal cost normalized to zero, the objective function $([P])$, also not defined in $\mathrm{CN}$, is equivalent to total profit divided by the number of Type 1 consumers, i.e., multiplying $[P]$ by $n 1$ results in a profit function that is further described in our Section 3. CN also define $\widehat{q}$ as the quantity where $v_{1}(\widehat{q})=v_{2}(\widehat{q})$ (see Proposition 1, p. 209).

\subsection{CN's Proposition 2}

CN's Proposition 2, found on p. 209, states:

(i) (The higher peak type gets efficient quantity) $q_{i}^{*}=q_{i}^{e}$, where $i=$ $\arg \max _{j} v_{j}\left(q_{j}^{e}\right)$.

(ii) (A sufficient and necessary condition for overall efficiency) $q_{i}^{*}=q_{i}^{e}$ and $t_{i}^{*}=v_{i}\left(q_{i}^{e}\right)(i=1,2)$ if and only if $v_{i}\left(q_{i}^{e}\right) \geq v_{j}\left(q_{i}^{e}\right)(i, j=1,2$ and $i \neq j)$.

As our concern is with the second part of this proposition, the following provides CN's proof of (ii).

(ii) (if Part) When $v_{i}\left(q_{i}^{e}\right) \geq v_{j}\left(q_{i}^{e}\right)(i, j=1,2$ and $i \neq j)$, offering a menu $T=\left\{\left(q_{i}^{e}, v_{i}\left(q_{i}^{e}\right)\right)\right\}(i=1,2)$ satisfies all constraints and extract all surplus from consumers.

(Only if Part) When $q_{i}^{*}=q_{i}^{e}$ and $t_{i}^{*}=v_{i}\left(q_{i}^{e}\right)(i=1,2)$, this part follows from $I C_{i}(i=1,2)$

\subsubsection{Our Argument with Proposition 2(ii)}

We will claim that (ii) is equivalent to the following: maximize the profit for each type separately (which is straightforward) and then check if the ICs hold. If they hold, the quantities form the solution. 
To elaborate, optimization for the firm occurs when quantity for each type corresponds to marginal cost (as $\mathrm{CN}$ normalize the cost to zero, it is $q_{i}^{e}$ ), and the firm captures the entire consumer surplus, i.e., $t_{i}^{*}=v_{i}\left(q_{i}^{e}\right), i \in\{1,2\}$. It remains to be determined whether the $I C$ s hold. However, the condition under Proposition 2 that $v_{i}\left(q_{i}^{e}\right) \geq v_{j}\left(q_{i}^{e}\right)$ is exactly the $I C$ when cost normalizes to zero. To see our claim, reduce both sides of the inequality with the price $t_{i}^{*}$ so that: $v_{i}\left(q_{i}^{e}\right)-t_{i}^{*} \geq v_{j}\left(q_{i}^{e}\right)-t_{i}^{*}$ or $0 \geq v_{j}\left(q_{i}^{e}\right)-t_{i}^{*}$. If Type $j$ deviates to the quantity of Type $i$, then his gain is negative. This negative inequality is not more restrictive than the $I C$. Consider that the gain of Type $j$ from his quantity $q_{j}^{e}$ is zero as $t_{j}^{*}=v_{j}\left(q_{j}^{e}\right)$. Substitute the zero on the left-side of the previous inequality so that $v_{j}\left(q_{j}^{e}\right)-t_{j}^{*} \geq v_{j}\left(q_{i}^{e}\right)-t_{i}^{*}$. Therefore, $v_{i}\left(q_{i}^{e}\right) \geq v_{j}\left(q_{i}^{e}\right)$ is equivalent to $v_{j}\left(q_{j}^{e}\right)-t_{j}^{*} \geq v_{j}\left(q_{i}^{e}\right)-t_{i}^{*}$ or $I C_{j}\left(I C_{i}\right.$ in $\left.\mathrm{CN}\right)$.

Under established mathematical practice, if the objective function is separated by some parameters but some of the constraints are not, one can solve for each set of parameters that are separated in the objective function, and then check if the mixed constraints hold. Consequently, $\mathrm{CN}^{\prime}$ s short proof on their p. 209 states only that the constraints hold and it is left to the reader to determine whether their claim to "provide a simple necessary and sufficient condition for overall efficiency" is actually informative. In fact, the following section illustrates that normalizing cost to zero greatly simplifies the $I C$ s but the simplification may lead to an anomaly.

\subsection{CN's Proposition 3}

For Proposition 3, $\mathrm{CN}$ introduce two general quadratic evaluation functions: $v_{1}(q)=q \cdot(1-b \cdot q / 2)$ and $v_{2}(q)=q \cdot(\alpha-b \beta \cdot q / 2)$, that are equivalent to two linear inverse demands: $p_{1}(q)=1-b \cdot q$ and $p_{2}(q)=\alpha-b \beta \cdot q$. For the two demands to cross, they restrict $0<\beta<\alpha<1$, and derive $q_{1}^{e}=1 / b<q_{2}^{e}=\alpha / b \beta, v_{1}\left(q_{1}^{e}\right)=1 /(2 b)$ and $v_{2}\left(q_{2}^{e}\right)=\alpha^{2} /(2 b \beta)$. The $v_{i}$ functions cross once at $\widehat{q}=\frac{2}{b} \frac{1-\alpha}{1-\beta}$.

CN's Proposition 3, found on p. 210, states:

(i) (Who gets efficient quantity) If $\alpha^{2} \leq \beta$ then $q_{1}^{*}=q_{1}^{e}$; if $\alpha^{2} \geq \beta$ then $q_{2}^{*}=q_{2}^{e}$.

(ii) (Overall efficiency) $q_{i}^{*}=q_{i}^{e}$ and $t_{i}^{*}=v_{1}\left(q_{i}^{e}\right) i=1,2$ if and only if $q_{1}^{e} \leq \widehat{q} \leq q_{2}^{e}$.

(iii) (Oversizing) $q_{1}^{*}=q_{1}^{e}$ and $q_{2}^{*}>q_{2}^{e}$ if and only if $q_{1}^{e}<q_{2}^{e}<\widehat{q}$.

(iv) (Undersizing) $q_{1}^{*}<q_{1}^{e}$ and $q_{2}^{*}=q_{2}^{e}$ if and only if $\widehat{q}<q_{1}^{e}<q_{2}^{e}$. 
As we will introduce an example that relates only to part (ii) of this proposition, the proof of parts (i) and (ii) follow directly from the corresponding parts of Proposition 2.

\subsubsection{Our Argument with Proposition 3(ii)}

We claim that Proposition 3(ii) can produce an example that defies economic sense. Begin with footnote 7 in CN's Conclusion: "Many convenience stores located along the US interstate highways (catering primarily to drivers on the go) charge more for a smaller cup of coffee than the larger one" (pp. 212-213). In the example we introduce below that is based on the $\mathrm{CN}$ model, the firm sets a price of 2 for 4 units and 1.25 for 10 units. We also explain how this paradox can be created in a simple $\mathrm{CN}$ model that includes $I C$.

We concentrate on CN's part (ii) above: (Overall efficiency) $q_{i}^{*}=q_{i}^{e}$ and $t_{i}^{*}=v_{i}\left(q_{i}^{e}\right), i=1,2$ if and only if $q_{1}^{e} \leq \widehat{q} \leq q_{2}^{e}$.

Consider first the case where $c=0$. Let $p_{1}(q)=1-0.25 \cdot q$ and $p_{2}(q)=0.25-0.025 \cdot q$, so that $b=0.25, \alpha=0.25, \beta=0.1$. It is easy to see that their condition is satisfied.

$$
\begin{aligned}
q_{1}^{e} & \leq \widehat{q} \leq q_{2}^{e} \quad \text { as } q_{1}^{e}=1 / b=4 \text { and } q_{2}^{e}=\alpha / b \beta=10 \\
4 & \leq \widehat{q} \leq 10 \quad \text { as } \widehat{q}=\frac{2}{0.25}\left(\frac{1-0.25}{1-0.1}\right)=6 \frac{2}{3}
\end{aligned}
$$

It is also easy to verify (see Figure 1) that the prices are: $T_{1}=t_{1}=v_{1}\left(q_{1}^{e}\right)=2$ for $q_{1}=4$, and $T_{2}=t_{2}=v_{2}\left(q_{2}^{e}\right)=1.25$ for $q_{2}=10$.

The utility of Type 1 equals the utility of Type 2 at $\widehat{q}=6 \frac{2}{3}$ meaning that it is in the negative price territory for Type 1 . The solution to the puzzle is that these prices will not be mathematically correct unless there is an increasing disposal cost at a rate of 0.25 per unit for Type 1 so that $\widehat{q}=6 \frac{2}{3}$ with total disposal cost of 0.89 . At $\widehat{q}, v_{1}\left(6 \frac{2}{3}\right)=v_{2}\left(6 \frac{2}{3}\right)=1.11$.

This example shows that CN's Proposition 3(ii) depends on the inverse demand function for Type 1 as having the same slope in the negative price region. CN's deviation from conventional practice explains why Type 1's IC is so easily mathematically satisfied.

The coffee example, previously described as part of the long footnote 7 in their Conclusion on pp. 212-213 suggests that $\mathrm{CN}$ were aware of this abnormality. Although we have not observed this phenomenon for the same product, e.g., regular brewed coffee as opposed to regular vs. espresso, one 


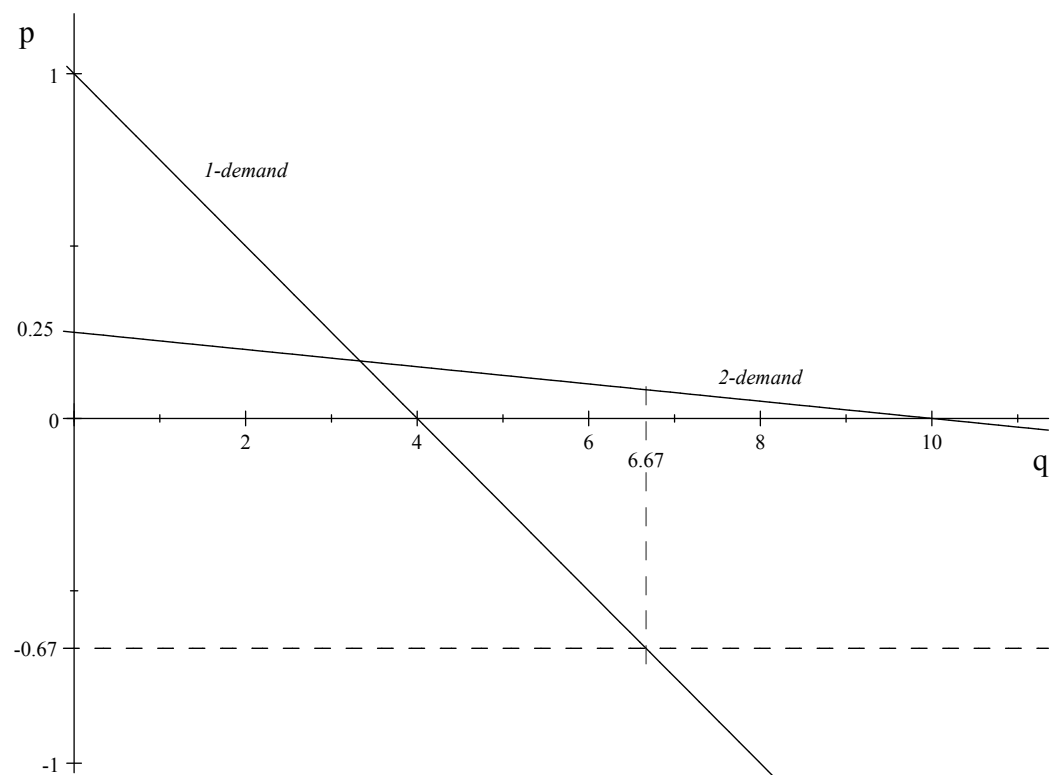

Figure 1: Valuations for the two consumers are equal at 6.67 units.

can reasonably assume that the disposal cost of coffee is zero. ${ }^{3}$ Thus, when the negative territory is excluded, the theoretical solution will be harder but more relevant to most economic applications.

We argue that the $\mathrm{CN}$ model is even more limited. By normalizing the cost to be zero, their model cannot distinguish between the negative and positive regions. To illustrate, let the cost of production in our example be $c=0.1$. It is easy to show that the prices of 4 and 10 units are 2.4 and 2.25 respectively. The anomaly remains. ${ }^{4}$ However when $c=1$, the anomaly disappears ( 6 for 4 units and 11.25 for 10 units).

3 CN claim that there are "many" such pricing schemes, but the prices of the smaller and larger quantities in the two additional examples described in their footnote 7 are the same. We agree that such cases are not unusual but CN's two additional examples do not fit their model or explain the anomaly.

4 As $\mathrm{CN}$ derive Proposition 3 specifically for two crossing linear demand curves, this anomaly will also apply to Proposition 2 that deals with general demand functions. 


\section{RESULTS UNDER FREE DISPOSAL}

Consider CN's model only for their case where the marginal cost is zero, i.e., $c=0$, as this will allow us to maintain the previous notation. The model has two crossing linear demand functions: $p_{1}=1-b q$ for the Type 1 consumers and $p_{2}=\alpha-b \beta q$ for the Type 2 consumers, where $0<\beta<\alpha<1$ (see Figure 2). There are $n_{t}$ Type $t$ consumers, $t \in\{1,2\}$.

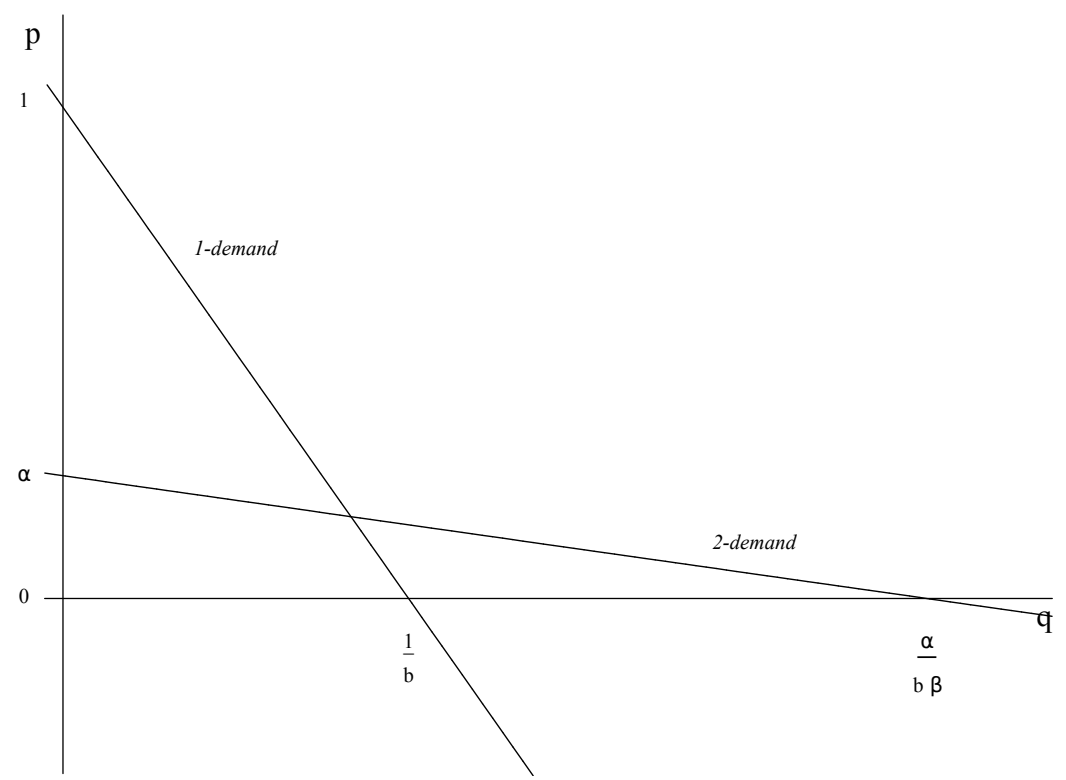

Figure 2: The model has two crossing linear demand functions: $p_{1}=1-b q$ for the Type 1 consumers and $p_{2}=\alpha-b \beta q$ for the Type 2 consumers, where $0<\beta<\alpha<1$.

$\mathrm{CN}$ provide valuation functions, $v_{1}(q)=q(1-b q / 2)$ and $v_{2}(q)=q(\alpha-$ $b \beta q / 2)$, that will correspond to utility when $c=0$. If we are restricted to positive prices (i.e., no disposal costs), then $v_{1}^{+}(q)$ and $v_{2}^{+}(q)$ can be defined as:

$$
\begin{gathered}
v_{1}^{+}(q)= \begin{cases}v_{1}(q)=q\left(1-\frac{b q}{2}\right) & \text { if } q<\frac{1}{b} \\
v_{1}\left(\frac{1}{b}\right)=\frac{1}{2 b} & \text { if } q \geq \frac{1}{b}\end{cases} \\
v_{2}^{+}(q)= \begin{cases}v_{2}(q)=q\left(\alpha-\frac{b \beta q}{2}\right) & \text { if } q<\frac{\alpha}{b \beta} \\
v_{2}\left(\frac{\alpha}{b \beta}\right)=\frac{\alpha^{2}}{2 b \beta} & \text { if } q \geq \frac{\alpha}{b \beta}\end{cases}
\end{gathered}
$$


Under the Revelation-Principle (Myerson, 1979), for any Bayesian equilibrium defined by a game, there exists a Bayesian equilibrium of an incentive compatible mechanism that will yield the same payoff. We can thus concentrate only on the case where the seller offers menu prices $T\left(q_{t}\right)$ for the quantities $q_{t}$, $t \in\{1,2\}$.

Let $v_{t}^{+}\left(q_{j}\right)$ be the utility of Type $t$ buyers from consuming $q_{j} ; t \in\{1,2\}$, $j \in\{1,2\}$. The firm's objective function is to maximize its profit subject to individual rationality constraints and the incentive compatibility constraints.

$$
\begin{array}{cll}
\max _{T\left(q_{t}\right)} & \pi=n_{1} \cdot T\left(q_{1}\right)+n_{2} \cdot T\left(q_{2}\right) & \\
\text { s.t. } & v_{1}^{+}\left(q_{1}\right)-T\left(q_{1}\right) \geq 0 & \mathrm{IR}_{1} \\
& v_{2}^{+}\left(q_{2}\right)-T\left(q_{2}\right) \geq 0 & \mathrm{IR}_{2} \\
& v_{1}^{+}\left(q_{1}\right)-T\left(q_{1}\right) \geq v_{1}^{+}\left(q_{2}\right)-T\left(q_{2}\right) & \mathrm{IC}_{1} \\
& v_{2}^{+}\left(q_{2}\right)-T\left(q_{2}\right) \geq v_{2}^{+}\left(q_{1}\right)-T\left(q_{1}\right) & \mathrm{IC}_{2}
\end{array}
$$

where $\pi$ represents the profit as the cost function is linear and marginal cost $c=0$. As before, $I R_{i}$ are the individual rationality constraints, and $I C_{i}$ are the incentive compatibility requirements, $i \in\{1,2\}$.

Following the arguments described in Section 2.2.1, to maximize $\pi$, the firm should maximize the profit for each type and then check if the $I C$ s hold. The firm should thus set the quantity for each type that corresponds to marginal cost which here is $0\left(\mathrm{CN}\right.$ label these quantities as $\left.q_{i}^{e}\right)$. This strategy enables the firm to capture the maximum consumer surplus from both types at quantities, $q_{1}^{e}=\frac{1}{b}$ and $q_{2}^{e}=\frac{\alpha}{b \beta}$ with prices $T\left(\frac{1}{b}\right)=v_{2}^{+}\left(\frac{1}{b}\right)=1 /(2 b)$ and $T\left(\frac{\alpha}{b \beta}\right)=v_{2}^{+}\left(\frac{\alpha}{b \beta}\right)=\alpha^{2} /(2 b \beta)$. As $T\left(q_{i}^{*}\right)=v_{i}^{+}\left(q_{i}^{*}\right)$, the first two constraints $\left(I R_{1}\right.$ and $\left.I R_{2}\right)$ are satisfied. It remains to be determined for which values of $b, \alpha$ and $\beta$ the ICs hold. The following develops an alternative to CN's Proposition 3(ii).

\section{Proposition}

If $2 \alpha-1 \leq \beta \leq \alpha^{2}$, then $q_{1}^{*}=q_{1}^{e}=1 / b$ and $q_{2}^{*}=q_{2}^{e}=\alpha /(b \beta)$ are the two menu quantities together with prices $T\left(\frac{1}{b}\right)=1 /(2 b)$ and $T\left(\frac{\alpha}{b \beta}\right)=\alpha^{2} /(2 b \beta)$, the firm's profits are maximized and the consumer surplus is zero.

Proof Let us start with $I C_{1}$, namely $v_{1}^{+}\left(q_{1}^{*}\right)-T\left(q_{1}^{*}\right) \geqq v_{1}^{+}\left(q_{2}^{*}\right)-T\left(q_{2}^{*}\right)$. As $T\left(q_{i}^{*}\right)=v_{i}^{+}\left(q_{i}^{*}\right)$, the left-hand side is 0 . It is left to show that $0 \geqq$ $v_{1}^{+}\left(q_{2}^{*}\right)-T\left(q_{2}^{*}\right)$, or $0 \geqq v_{1}^{+}\left(\frac{\alpha}{b \beta}\right)-T\left(\frac{\alpha}{b \beta}\right)$. As $v_{1}^{+}\left(\frac{\alpha}{b \beta}\right)=\frac{1}{2 b}$ and $T\left(\frac{\alpha}{b \beta}\right)=\frac{\alpha^{2}}{2 b \beta}$, then $0 \geqq \frac{1}{2 b}-\frac{\alpha^{2}}{2 b \beta} \Longrightarrow \frac{\alpha^{2}}{\beta} \geqq 1$. Therefore, $I C_{1}$ requires that $\alpha^{2} \geqq \beta$. 
For $I C_{2}, v_{2}^{+}\left(q_{2}^{*}\right)-T\left(q_{2}^{*}\right) \geqq v_{2}^{+}\left(q_{1}^{*}\right)-T\left(q_{1}^{*}\right)$. Again, the left-hand side is 0 so that $0 \geqq v_{2}^{+}\left(q_{1}^{*}\right)-T\left(q_{1}^{*}\right)$, or $0 \geqq v_{2}^{+}\left(\frac{1}{b}\right)-T\left(\frac{1}{b}\right)$. As $v_{2}^{+}\left(\frac{1}{b}\right)=(q \cdot(\alpha-b \beta \cdot q / 2))$ at $q=\frac{1}{b} \Longrightarrow v_{2}^{+}\left(\frac{1}{b}\right)=\frac{\alpha}{b}-\frac{\beta}{2 b}$ and $T\left(\frac{1}{b}\right)=\frac{1}{2 b}$, this leads to $0 \geqq\left(\frac{\alpha}{b}-\frac{\beta}{2 b}\right)-\frac{1}{2 b}$, or $0 \geqq 2 \alpha-\beta-1$. Therefore, $I C_{2}$ requires that $\beta \geqq 2 \alpha-1$.

Note that the proposition is independent of $b$ as $\mathrm{CN}$ were able to normalize the quantity, namely, $b=1$. Therefore, $q_{1}^{*}=q_{1}^{e}=1$ and $q_{2}^{*}=q_{2}^{e}=\frac{\alpha}{\beta}$ with respective prices $T(1)=0.5$ and $T\left(\frac{\alpha}{\beta}\right)=\alpha^{2} /(2 \beta)$.

To more clearly illustrate the role of the $I C \mathrm{~s}$, consider extreme values of $\alpha$, for example, 0.1 and 0.9 . If $\alpha=0.1$, then $0 \leq \beta \leq 0.01$. Choosing $\beta=0.005$ leads to $q_{2}^{*}=20$ and $T(20)=1$, i.e., the quantity for Type 2 is 20 times that for Type 1 . With $\alpha=0.1,2 \alpha-1$ is negative so that $I C_{2}$ becomes irrelevant and $I C_{1}$ is the only binding constraint. Consistent with intuition, the price of the larger quantity must be higher than the price of the smaller one (otherwise Type 1 will buy the larger quantity and dispose any amount above 1$)$. As $\alpha=0.1$, and the price for Type 2 must be higher, $T\left(q_{2}^{*}\right)=v_{i}\left(q_{2}^{*}\right)=T\left(\frac{\alpha}{\beta}\right)=\frac{\alpha^{2}}{2 \beta} \geq T\left(q_{1}^{*}\right)=\frac{1}{2}$, it follows that $\beta$ must be small, i.e., $\frac{\alpha^{2}}{2 \beta} \geq \frac{1}{2}$.

If $\alpha=0.9$, then $0.8 \leq \beta \leq 0.81$. Choosing $\beta=0.805$, it follows that $q_{2}^{*}=1.118$ and $T(1.118) \simeq 0.503$. Unlike the previous example, both $I C \mathrm{~s}$ determine the range of $\beta$. The condition $I C_{2}(2 \alpha-1 \leq \beta)$ determines that the utility of Type 1 is higher than the utility of Type 2 from the smaller quantity $q_{1}^{*}=q_{1}^{e}=1$. Therefore, $\beta$ must be large as the utility of Type 1 is $v_{1}^{+}(1)=T(1)=\frac{1}{2}$, while the utility of Type 2 is $v_{2}^{+}(1)=q \cdot(\alpha-b \beta \cdot q / 2)=$ $1 \cdot(0.9-1 \cdot 0.805 \cdot 1 / 2)=0.4975$. Similar to the previous example, $I C_{1}$ will require that the price of $q_{2}^{*}=1.118$ must be more than $T(1)=0.5$. Therefore, the price $v_{2}^{+}(1.118)=T(1.118) \simeq 0.503$.

Figure 3 shows the two $I C$ constraints and the non-negative region between them, labelled as $A$, where $\beta$ satisfies: $\operatorname{Max}\{0,2 \alpha-1\} \leq \beta \leq \alpha^{2}$.

As seen in Figure 3, with $\alpha=0.5$, the condition $(2 \cdot \alpha-1)=0 \leq \beta \leq$ $0.25=\alpha^{2}$ will allow $\beta$ to have larger ranges between the constraints $I C_{1}$ and $I C_{2}$. In contrast, as we have explained using $\alpha=0.1$ (which is far from the intercept of Type 1's demand function in Figure 2), the slope of Type 2's demand function is much more horizontal than that of Type 1, and the profit maximizing quantities will vary greatly between the types. On the other hand, when $\alpha=0.9$, which is near the intercept for Type 1's demand, the slopes of the two demands are similar, and the profit maximizing quantities will be close. 


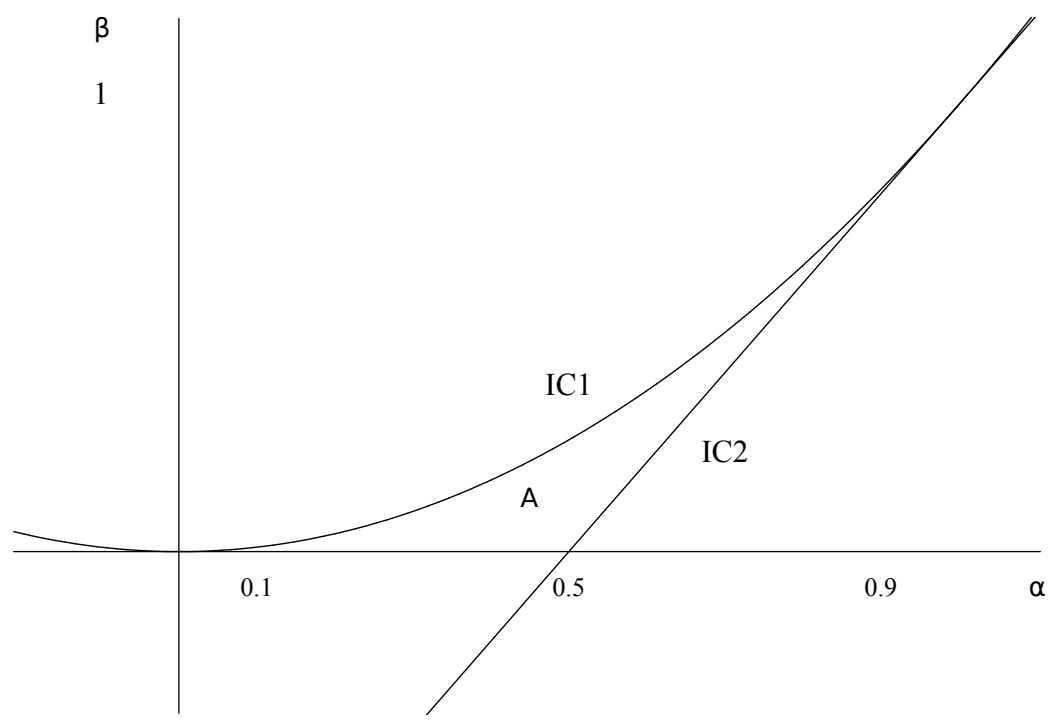

Figure 3: The region A where the incentive compatibility constraints are satisfied.

\section{CONCLUSION}

Price discrimination, especially in the fast food sector, is of considerable academic and policy interest. Scholars across a range of disciplines have focused on the pricing, marketing and health effects of supersized portions of "junk food". ${ }^{5}$ Economic advances in our understanding of this "value-size pricing" through models of price discrimination should, in principle, contribute to the development of more effective policy and regulatory actions to limit consumption of such products.

CN's theoretical approach appears to provide new insights by easily solving the nonlinear tariff challenge for general utility functions. However, given their normalization of cost to zero, their condition for Proposition 2(ii) is just

5 "Junk food" is often associated with calorie-dense food that has high levels of sugar, glycemic starch, and saturated fat. Vermeer, Steelhuis, and Vermeer et al. (2014) summarize some of the policy interventions to reduce portion sizes. As for strategies where firms charge the same price for any sized beverage (see our footnote 3), Haws et al. (2020) recently examined the effects of this strategy on consumption of soft drinks. In addition to increasing beverage size choices compared to standard pricing and, unlike standard pricing, this strategy nullifies the effectiveness of calorie postings in reducing larger sizes. 
a variant of the $I C$. CN's subsequent restriction to linear demand functions that violate the SCC, while failing to exclude the negative price region, may seriously limit the economic relevance of Propositions 2 and 3. Analyses involving violations of the SCC should not be considered as settled and, as such, further research for these cases is well warranted.

\section{References}

Andersson, T. (2005). Profit maximizing nonlinear pricing. Economics Letters, 88(1), 135-139.

Andersson, T. (2008). Efficiency properties of non-linear pricing schedules without the single-crossing condition. Economics Letters, 99(2), 364-366.

Chao, Y., \& Nahata, B. (2015). The degree of distortions under second-degree price discrimination. Economics Letters, 88(1), 135-9.

Haws, K., Liu, P., Dallas, S., Cawley, J., \& Roberto, C. (2020). Any size for a dollar: The effect of any-size-same-price versus standard pricing on beverage size choices. Journal of Consumer Psychology, 30(2), 392-401.

Maskin, E., \& Riley, J. (1984). Monopoly with incomplete information. RAND Journal of Economics, 15(2), 171-196.

Myerson, R. (1979). Incentive-compatibility and the bargaining problem. Econometrica, 47(1), 61-73.

Tirole, J. (1988). The Theory of Industrial Organization. Cambridge: MIT Press.

Vermeer, W., Steenhuis, I., \& Poelman, M. (2014). Small, medium, large or supersize? The development and evaluation of interventions targeted at portion size. International Journal of Obesity, 38(1), 513-518. 